\title{
Augmenting Hardware Experiments with Simulation in Digital Communications
}

\author{
Dennis Silage \\ Electrical and Computer Engineering \\ College of Engineering, Temple University
}

\section{So Much Equipment, So Little Time}

An undergraduate course in digital communications is usually offered with a supplemental hardware laboratory to illuminate the concepts presented in the course text. The traditional undergraduate laboratory presents communication circuit hardware (phase-locked loops, voltagecontrolled oscillators) and systems (modulators, demodulators, filters) in the context of the measurements provided by complex instruments (modulation and spectrum analyzers, sweep frequency generators) and techniques (bandwidth, distortion and bit error) ${ }^{1}$. The operation of these instruments is often daunting to the undergraduate and somewhat out-of-context with the course text and with the computer-aided techniques employed in undergraduate laboratories offered in other course sequences, such as electronics, control systems, and digital logic.

A reasonable solution to this dilemma is to provide an undergraduate communications laboratory with a computer data acquisition and interactive process control system, such as LabView ${ }^{2}$. Another modern, fully interactive, computer-aided digital communication hardware laboratory is Discovery II by Feedback (www.feedback.com), which provides theory and background, a measurement practicum, and probing questions. Virtual measurement techniques are employed using a custom high-speed (USB) data acquisition and control system interface to hardware modules and interactive references and graphics. The student is required to obtain, analyze and interpret the measurements observed at various points in the communication system.

Although such a communication hardware laboratory provides the student with a firm basis for understanding and an exposure to temporal and spectral measurement techniques, they are confined to the expanse of the hardware. These hardware laboratory experiments also become highly repetitive with yearly use. Augmenting these fundamental but fixed experiments with innovative simulations, prepared new each semester, provides an environment for understanding with material that is both stimulating and non-repetitive in content. The simulation experiments do not replace the hardware measurement practicum here, but rather extend and enhance them.

Communication systems convey information from a transmitter over a channel to a receiver. Modern communication systems do so in the presence of additive noise and mild to severe system non-linearity, which tend to corrupt the transmission. Examining the performance of a communication system as a set of analytical expressions, even if random noise and system nonlinearities can be described adequately, seems to provide little insight or motivation ${ }^{3}$. However, 
simulation can provide such motivation by allowing the student to try the what-ifs of communication system design ${ }^{4}$.

\section{Simulation Using a Sound Environment}

SystemView by Elanix (www.elanix.com) is a graphical simulation environment for baseband and passband communication systems. Although SystemView has extensive professional capabilities that support digital signal processing and analog radio frequency design, it can be used to animate the typical block diagram representations of fundamental analog and digital communication systems. Temporal and spectral signal displays can illustrate both their ideal and degraded performance due to additive noise and system non-linearity. SystemView also has a sound capability utilizing .wav file for input and output that can be used to extend the analysis of the communication system to an aural assessment of performance using speech source files. Students can experience the degradation of performance of a communication system in a direct manner.

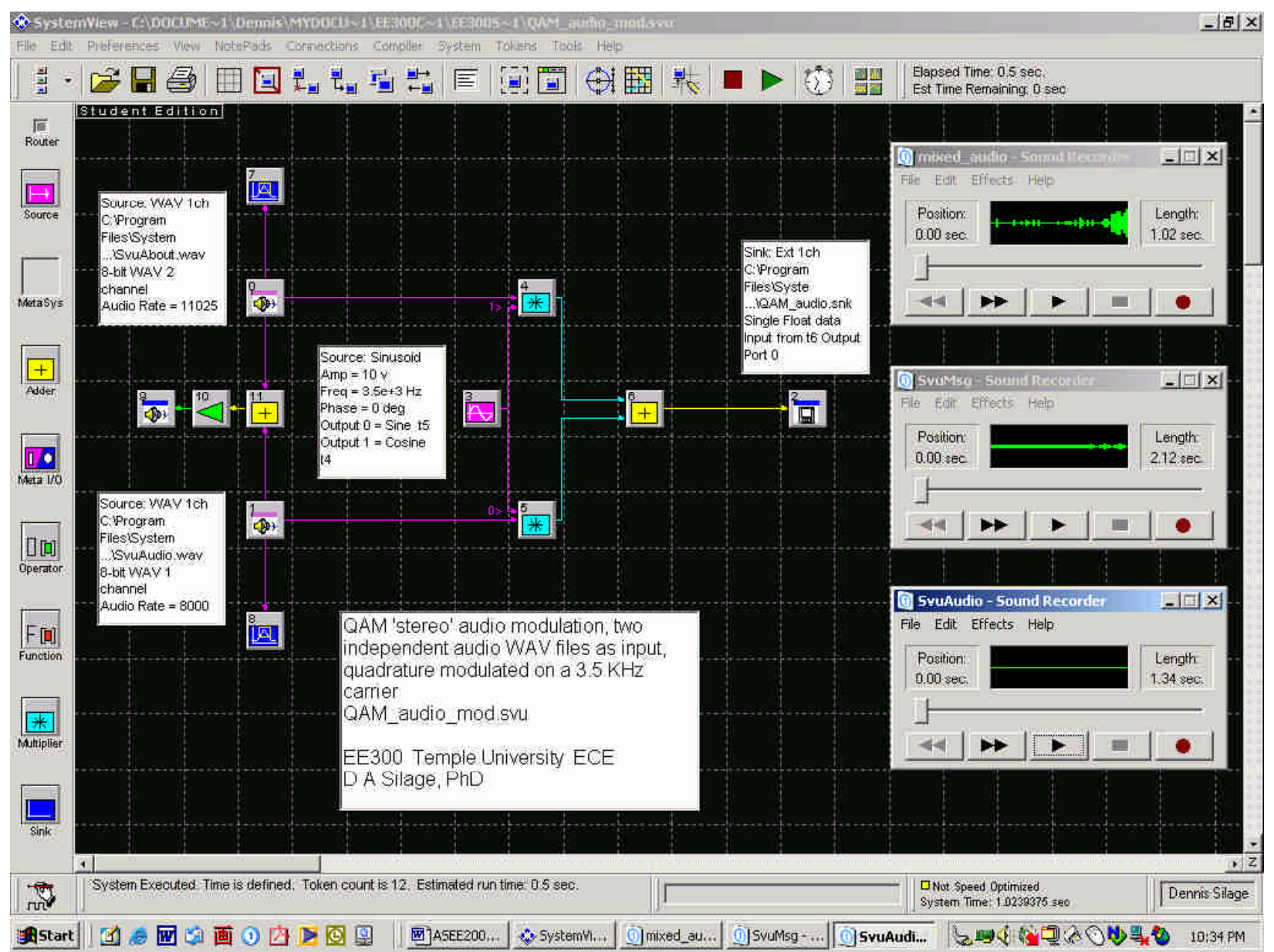

Figure 1. SystemView simulation of a stereo audio quadrature DSB-SC amplitude modulation.

The SystemView simulation environment consists of a System Window, as shown in Figure 1, and an Analysis Window, as shown in Figure 4. The System Window provides a standard Windows 
Menu, a Toolbar, horizontal and vertical Scroll Bars, a Design area, a Message area, and a Token Reservoir. The Analysis Window also provides a standard Windows Menu, a Toolbar, horizontal and vertical Scroll Bars, a Plot area for graphically displaying signals and data, a Message area, and a Sink Calculator. The online Elanix SystemView User's Guide gives a complete description of the Menu, Token Reservoir and Toolbar. Students are also provided with SystemView with Examples (course EE300 at http://blackboard.temple.edu) as a quick-start laboratory manual for the simulation environment. The simulations shown here can also be viewed at this course site.

An interesting introduction to capabilities of the SystemView simulation environment and with sound is an extension of an analog double sideband suppressed carrier (DSB-SC) amplitude modulation and demodulation system to a two channel audio demonstration, as shown in Figures 1 and 2. In Figure 1, two DSB-SC modulators (multipliers) have a .wav file as one input and a quadrature carrier signal as the other input. Speech .wav files can be recorded or played-back by the student using the Sound Recorder of the Windows environment, as shown in Figure 1.

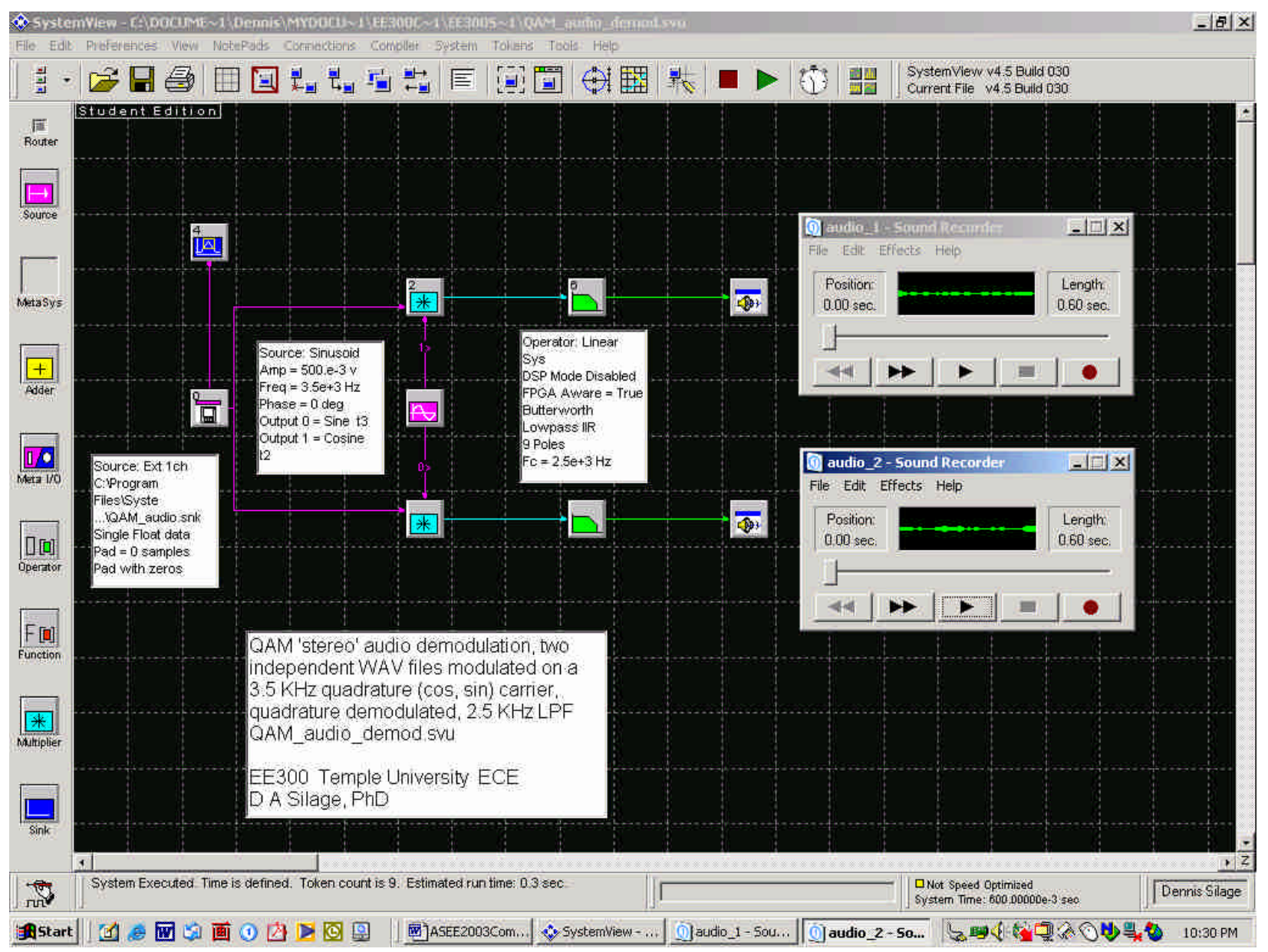

Figure 2. SystemView simulation of a stereo audio quadrature DSB-SC amplitude demodulation.

The quadrature carrier signals are generated by the same source token with concurrent sine and cosine outputs. Since the two audio . wav files are quite distinct, stereo audio quadrature DSB- 
SC amplitude modulation occurs. The two audio files can be played-back and the student can verify that they are in fact different. The resulting modulated signal is recorded in a single precision output file for demodulation, as shown in Figure 1.

Simple coherent quadrature carrier DSB-SC demodulation, without carrier recovery, is shown in Figure 2. The demodulated audio .wav files can again be played-back by the student using the Sound Recorder to verify the recovery of two channels of information. Although the temporal audio waveforms here can also be easily viewed in the SystemView Analysis Window, such a signal display is obviated by their more familiar aural presentation of the stereo separation provided by quadrature modulation.

An additive white Gaussian noise (AWGN), fading channel or carrier interference system can be inserted between the modulation and demodulation systems to demonstrate communication in a degraded environment to the student. Intentional errors in the frequency or phase of the quadrature carrier demodulator signals can illustrate an important issue in receiver performance. This simulation example and extensions can be used in the course lecture and demonstrates the educational versatility of SystemView. The salient concept is the interesting animation of the analytical expressions and block diagrams of a tradition undergraduate communication textbook provide by simulation.

\section{ASK, What You Can Do}

The premise here is that innovative simulation experiments augment the firm foundation provided by the hardware laboratory. An example of this augmentation from the current communication laboratory sequence (http://blackboard.temple.edu/EE300) is the amplitude shift keying (ASK) module presented here.

The Discovery II hardware laboratory in ASK is used to introduce the student to unipolar and bipolar non-return to zero (NRZ) binary lines codes, producing suppressed carrier and carrier ASK modulation. Non-coherent envelope and coherent demodulation of ASK is also investigated in the measurement practicum. The student performs these ASK hardware experiments first and then is presented with a SystemView ASK simulation example with a further investigation, new each semester, which cannot be accomplished with the fixed Discovery II hardware.

Figure 3 shows the SystemView System Window for a unipolar NRZ binary line code ASK modulator, as in the Discovery II ASK hardware laboratory, and Figure 4 is the resulting power spectral density (PSD) for a carrier frequency of $20 \mathrm{KHz}$ and a bit rate of $1 \mathrm{KHz}$. For a unipolar NRZ binary line code, a pronounced carrier component is observed. The SystemView simulation utilizes a pseudorandom noise (PN) binary source, while the Discovery II hardware has a finite shift register data source.

Figure 3 also shows the SystemView System Time Specification query, which introduces the student to the concept of spectral resolution in the discrete Fourier transform (DFT). The resulting SystemView ASK simulation provides a clear demonstration to the student that these spectral results are comparable to that observed in the Discovery II hardware experiments. 


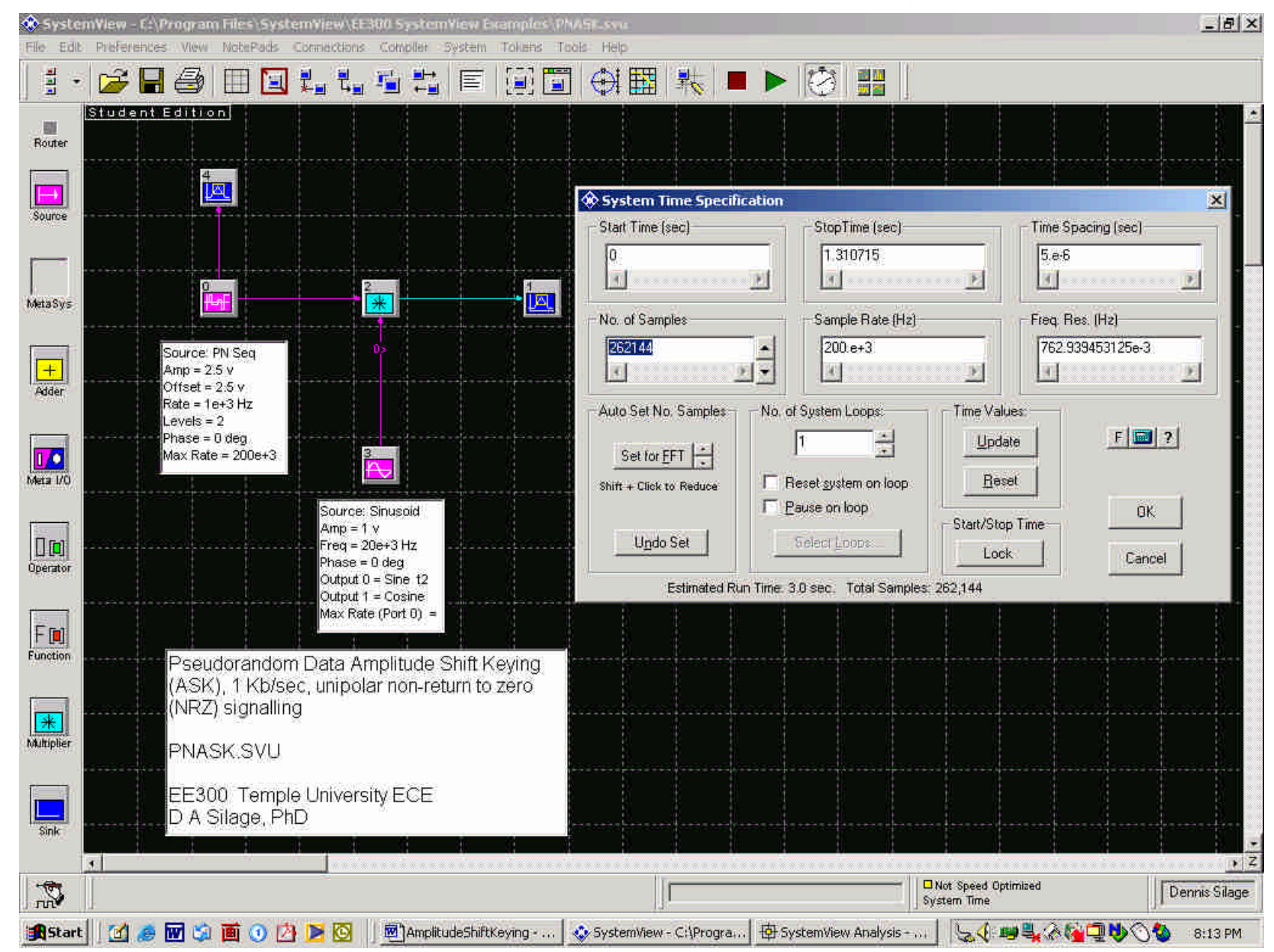

Figure 3. SystemView simulation of PN source, unipolar NRZ ASK data.

The first part of the SystemView ASK simulation experiment, new for this semester, requires that the student generate a bipolar return to zero (RZ) and an alternate mark inversion (AMI) binary line code from the PN binary, unipolar source token. These lines codes are not included as a part of the Discovery II ASK hardware experiment. SystemView provides a variety of tokens, including combination and sequential logic elements in the Logic Library, which are designed and configured by the student to provide these two new binary line codes from the PN source data. A prerequisite to this course in digital communication is the course in digital logic. The student reconfigures the simulation example to provide ASK modulation of these new binary line codes.

The student is then asked to describe the PSD of these new binary line codes and compare their form (presence of a carrier component, spectral sidebands, repetitive nulls) and proscribed bandwidth in a engineering report format to those observed in the Discovery II ASK hardware laboratory. The SystemView Analysis Window has an interactive measurement cursor that can be used to obtain these spectral measurements, as shown in Figure 4. The suppressed carrier component and the spectral nulls centered about the carrier frequency at multiples of the bit rate that the student observes in this augmented ASK simulation laboratory illuminate the material in the course text, which serves as a reference. 


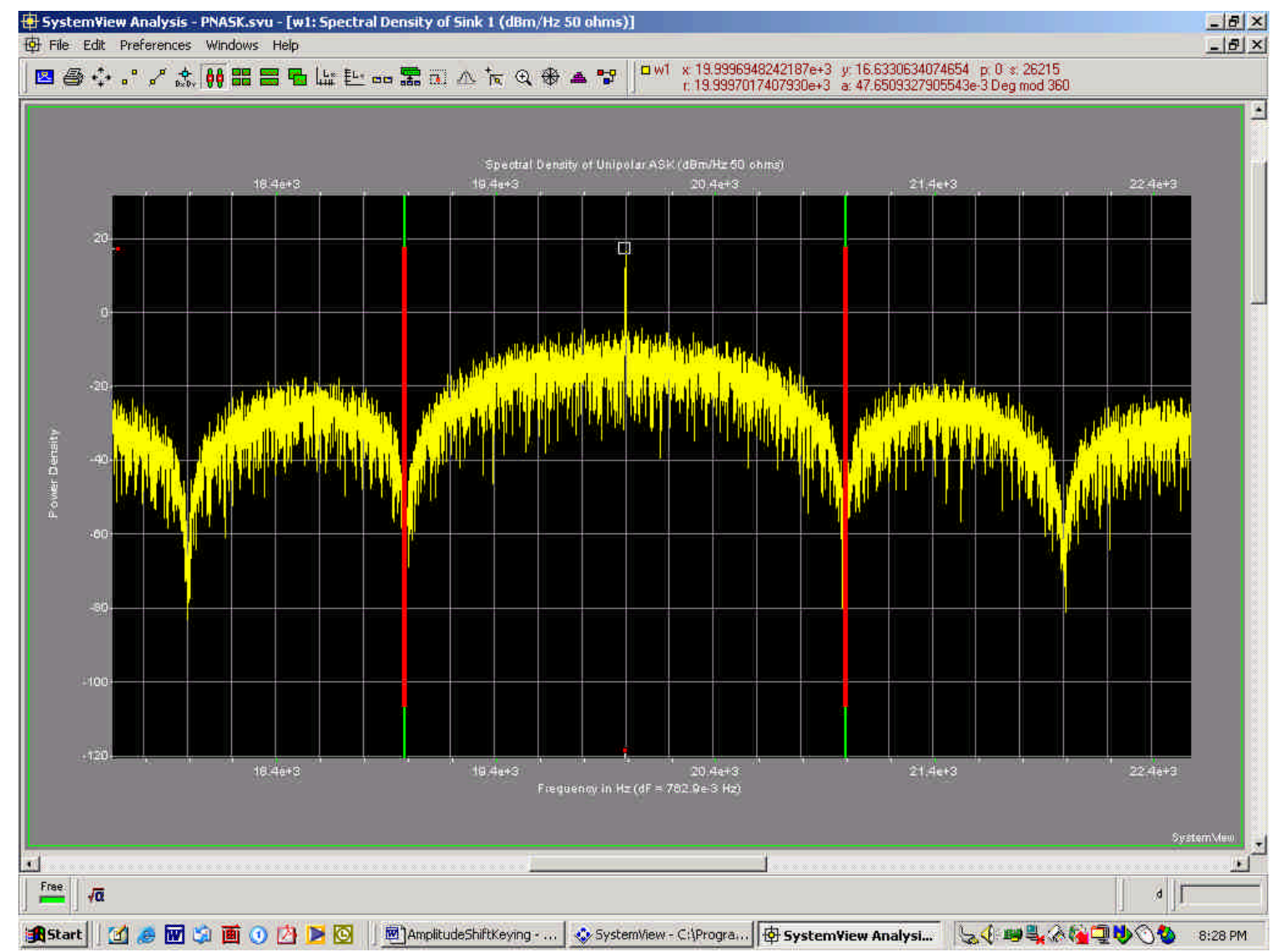

Figure 4. SystemView simulated PSD for a PN source, unipolar NRZ data ASK with a carrier frequency of $20 \mathrm{KHz}$ and a bit rate of $1 \mathrm{KHz}$.

The second part of the SystemView ASK simulation experiment is to implement the non-coherent envelope and coherent demodulation of ASK, as in Discovery II ASK hardware laboratory, using the source (oscillator), non-linear function (rectifier, multiplier) and linear system operator (filter) tokens available. An AWGN channel, implemented as a Gaussian noise source and adder tokens, introduces bit errors in the data transmission. The student then performs the requisite simulations and analyzes the resulting bit error rate (BER), comparing these results to the analytical expressions in the course text. SystemView provides a BER token in the Communications Library, which computes the cumulative average and total bit errors observed. Figure 5 is the SystemView System Window that shows a PN source with AWGN and the BER token, which is provided to the student as an example of this measurement.

\section{Keeping It Fresh}

Undergraduate laboratories require constant attention and formulation of new and innovation experiments each semester. Rather than adopt only a simulation ${ }^{4}$ or a complex hardware approach $^{5}$, a modern, interactive hardware digital communication laboratory here provides a firm foundation for an augmented series of experiments using simulation. However, there are two salient questions. 


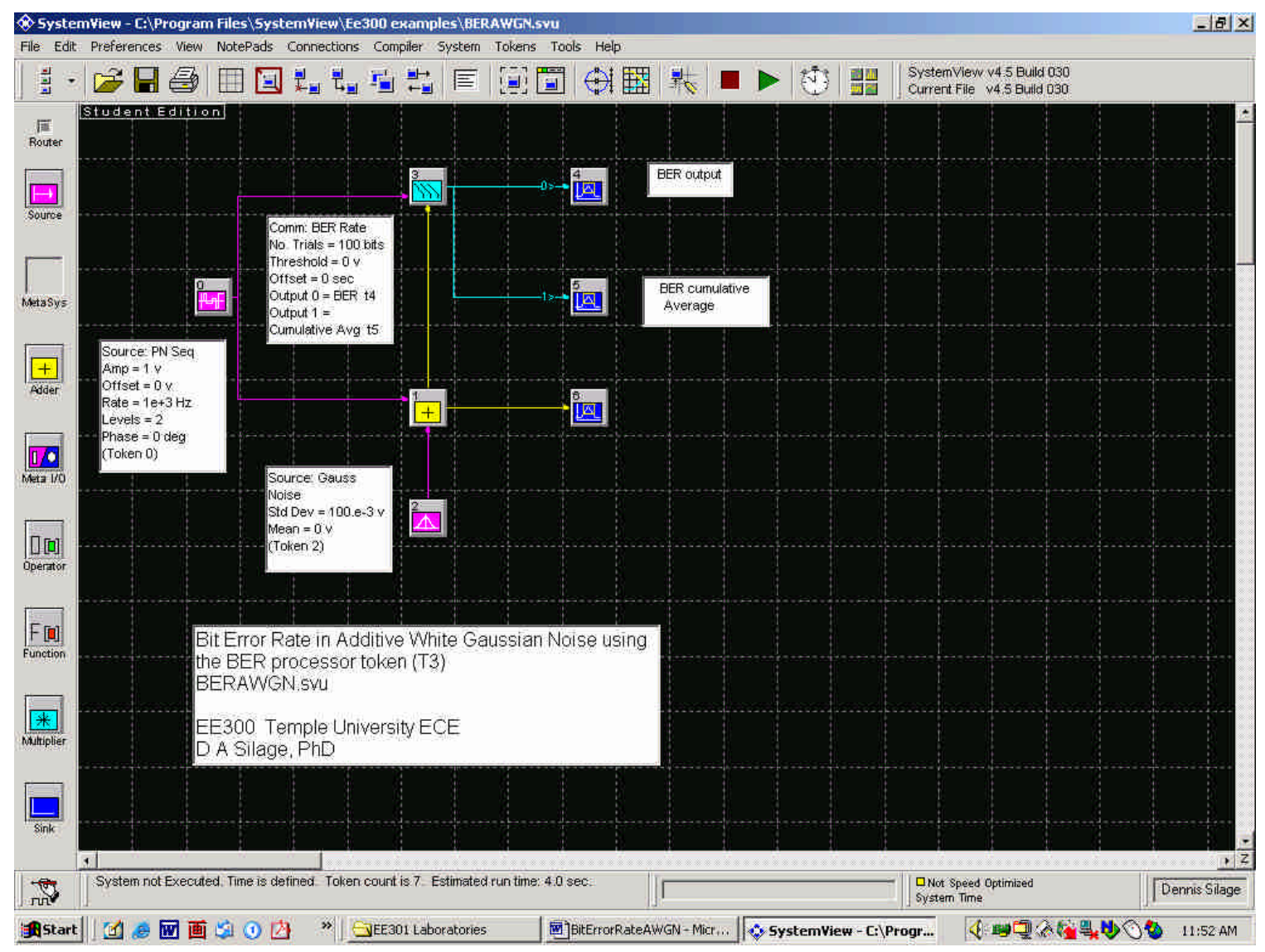

Figure 5. SystemView BER analysis of a PN source with AWGN.

Is the hardware laboratory a requisite? Although the simulation experiments can certainly replicated the observed temporal waveforms and resulting PSD of the hardware, undergraduate students require didactic materials in multiple formats to understand the concepts being presented. Discovery II provides that format and features matching test points on its hardware module for an auxiliary connection to an oscilloscope. If used, the oscilloscope measurement substantiates to the student that the data acquisition system and graphical display is functioning.

But can new digital communication simulation experiments be prepared for each semester? First, the PN source data rates, modulation sensitivities (V/V, Hz/V, and $\mathrm{rad} / \mathrm{V}$ ), and carrier frequency and amplitudes are varied each semester to change the form of the resulting PSD. Second, the simulation experiments focus on different aspects of the digital modulation and demodulation process each semester. Some examples of interesting simulation experiment modalities have included: errors induced by carrier frequency and phase jitter, demodulation using low-pass filters with insufficient bandwidth, incidental frequency modulation (FM) in coherent demodulation of ASK, very narrowband binary frequency shift keying (BFSK) and binary phase shift keying (BPSK), pulse code modulation (PCM) with stuck bits, extended binary line codes, and burst errors in digital communication. 
Simulation has been used in the undergraduate analog and digital communication curriculum for more than a decade. Prior to the introduction of SystemView by Elanix (1997), TESLA by Tesoft was used (1989-1996). SystemView is now also used in our graduate telecommunication and digital communication sequence. Because of the investigative nature in the simulation of these augmented digital communication laboratories, the undergraduate student is seemingly well prepared for advanced study.

\section{Assessment}

The undergraduate digital communications laboratory has used simulations to supplement the requisite hardware laboratory for well over a decade. Therefore, the efficacy of the use of simulation cannot be assessed directly. However, Departmental course feedback surveys are used to gauge the response of the student to this approach for the communications laboratory, with questions such as: "What do the hardware laboratory experiments teach you?" and "How do the simulation experiments help to explain the results observed in the hardware experiment?".

\section{Acknowledgement}

Elanix, Inc. (www.elanix.com) supports the use of advanced communication simulation software in undergraduate and graduate courses and research by providing SystemView to the academic community.

1. Kamali, B. Development of an Undergraduate Structured Laboratory to Support Classical and New Base Technology Experiments in Communications. IEEE Trans. Education, 37:1, pp. 97-100 (1994).

2. Keene, H. and Parten, S. Advanced Communication Test System. Proc. ASEE Annual Conf., Session 3220 (2001).

3. Jennison, B. and Kohne, G. A Coherent Theory/Hardware Course in Communication System Design. Proc. ASEE Annual Conf., Session 2625 (2000).

4. Kramer, K. Using MATLAB-based Laboratories to Demonstrate Wireless Communication System Principles. Proc. ASEE Annual Conf., Session 2793 (2001).

5. Ofosu, W., Garner, J. and Metz, D. Laboratory Exercise on Demodulation of a PAM Signal. Proc. ASEE Annual Conf., Session 1426 (2001).

DENNIS SILAGE received the $\mathrm{PhD}$ in Electrical Engineering from the University of Pennsylvania in 1975. He is a Professor, teaches digital communication and digital signal processing, and is the trustee of the Temple University Amateur Radio Club K3TU (http://www.temple.edu/k3tu), which he has integrated into the undergraduate communications curriculum. Dr. Silage is a past chair of the Mid Atlantic Section of the ASEE. 